\title{
Minimal gravity and Frobenius manifolds: bulk correlation on sphere and disk
}

\author{
Konstantin Aleshkin, ${ }^{a, b}$ Vladimir Belavin ${ }^{c, 1}$ and Chaiho $\mathbf{R i m}^{d}$ \\ ${ }^{a}$ L.D. Landau Institute for Theoretical Physics, \\ Akademika Semenova av., 1-A, Chernogolovka, Moscow Region, 142432 Russia \\ ${ }^{b}$ International School for Advanced Studies (SISSA), \\ via Bonomea 265, Trieste, 34136 Italy \\ ${ }^{c}$ Department of Particle Physics and Astrophysics, Weizmann Institute of Science, \\ Rehovot, 7610001 Israel \\ ${ }^{d}$ Department of Physics, Sogang University, \\ Seoul, 121-742 Korea \\ E-mail: kkcnst@gmail.com, belavin@lpi.ru, rimpine@sogang.ac.kr
}

ABSTRACT: There are two alternative approaches to the minimal gravity — direct Liouville approach and matrix models. Recently there has been a certain progress in the matrix model approach, growing out of presence of a Frobenius manifold (FM) structure embedded in the theory. The previous studies were mainly focused on the spherical topology. Essentially, it was shown that the action principle of Douglas equation allows to define the free energy and to compute the correlation numbers if the resonance transformations are properly incorporated. The FM structure allows to find the explicit form of the resonance transformation as well as the closed expression for the partition function. In this paper we elaborate on the case of gravitating disk. We focus on the bulk correlators and show that in the similar way as in the closed topology the generating function can be formulated using the set of flat coordinates on the corresponding FM. Moreover, the resonance transformations, which follow from the spherical topology consideration, are exactly those needed to reproduce FZZ result of the Liouville gravity approach.

KEYwords: 2D Gravity, Matrix Models, Conformal Field Theory, Nonperturbative Effects

ARXIV EPRINT: 1708.06380

\footnotetext{
${ }^{1}$ Weston Visiting Professorship at Weizmann Institute. On leave from Lebedev Physical Institute, Moscow.
} 


\section{Contents}

1 Introduction 1

2 Dual approach to MLG 3

2.1 Douglas equation and Frobenius manifolds 3

2.2 Scaling dimensions and 2D gravity 4

$\begin{array}{lll}2.3 & \text { Bulk generating function on the disk } & 7\end{array}$

3 Unitary models $\quad 9$

$3.1 \mathrm{M}(3 / 4)$ : Ising model — the simplest unitary case $\quad 9$

$3.2 \mathrm{M}(\mathrm{q} / \mathrm{q}+1)$ : towards the general case $\quad 11$

4 Non-unitary models $\quad 14$

4.1 $\mathrm{M}(2 / 2 \mathrm{~s}+1)$ : Lee-Yang series 14

$\begin{array}{lll}4.2 \mathrm{M}(3 / 5) \text { : new features } & 17\end{array}$

$\begin{array}{lll}5 & \text { Concluding remarks } & 19\end{array}$

$\begin{array}{ll}\text { A Bessel functions and Chebyshev polynomials } & 20\end{array}$

\section{Introduction}

The conformal bootstrap solution of Liouville field theory (LFT) [1-4] leads to the possibility $^{1}$ to formulate the "direct" path integral approach to $c<1$ minimal string theory, known also as two-dimensional minimal Liouville gravity (MLG) [1]. Computations in this framework are rather complicated, since in all but simplest cases they require separate analysis of the correlation functions in both gravitational (LFT) and matter (Minimal Model) sectors [7], a careful treatment of the discrete terms [8] arising in the Liouville operator product expansion, integrating over moduli spaces of curves, etc. On the other hand, the "dual" matrix models (MM) approach [9-11], based on the discretization of the path integral and consequent double scaling limit, provides an efficient alternative description of $2 \mathrm{D}$ gravity and also reveals an integrable structure of the theory through the connection with a certain class of integrable hierarchies [12-15]. Different checks in 90s (see, e.g. [16-21]) pointed out that matrix models are connected with the minimal Liouville gravity if the connection is properly understood. More elaborate checks performed on the level of correlation functions confirmed this assumption, after the discovery of Liouville higher equations of motion [22] opened the way of analytic computations of the moduli integrals [23]. In particular, for the

\footnotetext{
${ }^{1}$ For recent development aimed to give a rigorous proof of the LFT construction and also of its integrable structure, see $[5,6]$.
} 
minimal gravity models of Lee-Yang series $M(2 / p)$, corresponding to one-matrix models, the explicit form of the resonance transformations form $\mathrm{KdV}$ time-parameters of MM to the Liouville coupling constants of MLG has been established in [24]. Further progress [2529] has been made after establishing the connection between the Douglas string equation approach [12] to the matrix models and the Frobenius manifolds [30]. This connection allowed to find explicitly the generating function of the correlation numbers for the general case of gravitational $M(q / p)$ minimal models. However, the consideration has been so far restricted to the case of gravitational models defined on closed Riemann-surfaces such as the sphere or the torus [31-34], relevant for the sector of closed strings.

In this paper, we are interested in the boundary minimal Liouville gravity (BMLG), relevant for open minimal strings. The worldsheet approach to BMLG requires analyzing boundary minimal models, LFT and ghosts and seems even more complicated compared to the closed case. In addition to the bulk MLG content, the classification of physical fields, or BRST cohomologies, in BMLG is specified by a set of boundary changing fields leading to admissible boundary conditions [35]. Constructing correlation numbers, even before taking moduli integrals, requires knowledge of the structure constants appearing in the operator product expansion of two bulk fields, the structure constants of boundary fields, the couplings between bulk and boundary fields and the one point functions of the identity operator for different boundary conditions [36-40].

In the dual approach the boundary effect was first considered in [21]. Since then, it has been investigated in many different contexts. One may refer to some of the previous studies in RSOS models and $\mathrm{O}(\mathrm{N})$ fluctuating models [41-43], in loop gas models [44, 45] and in (one-) [46] and (two-) [47, 48] matrix models. The boundary effect for the bulk correlation in the Lee-Yang series of the minimal gravity models was augmented by the resonance transformation in [49]. However, as mentioned above, the Lee-Yang series is represented by one matrix models and it turns out that the related FM is trivial (i.e. one-dimensional). Essentially, it means that the MM partition function found in [21] is easily translated to the MLG partition function, the only care is needed to properly take into account the resonance transformations. Other minimal models are given in terms of multi-matrix models, the corresponding Frobenius manifolds are multi-dimensional and higher Gelfand-Dickey hierarchies appear in general case [25, 27]. The analysis of the boundary effect on the MLG models related to multi-dimensional Frobenius manifolds is still missing. In particular, there is no closed expression for the BMLG generating function of bulk correlation numbers available yet. In this work, we extend the analysis of Frobenius manifolds for the bulk correlation on the disk.

The paper is organized as follows. In section 2, we recall the dual approach to MLG and provide the framework of finding bulk correlation numbers on the sphere and on the disk. It turns out that the role of the Douglas equation on the disk is slightly different from that on the sphere, where explicit dependence of the Frobenius (flat) coordinates on the Liouville couplings is required to be determined. On the other hand, the resonance transformation on the disk is the same as in the spherical topology. Even though this statement is not completely obvious, ${ }^{2}$ it is anticipated because the boundary operators

\footnotetext{
${ }^{2}$ The physical origin of the resonance transformations is the ambiguities coming from contact terms in the Euclidean correlators at the coincident points of the operators $[21,50]$.
} 
that could additionally contribute to the coupling mixing are not considered here [51]. In section 3, we consider the effect of presence of nontrivial Frobenius manifolds for the unitary series. We present the Ising model case $M(3 / 4)$ as an example. In section 4 we discuss non-unitary case. We start with the Lee-Yang series. Even though the Frobenius manifold is one-dimensional, the uniform FM description allows to represent the disk partition function as an integral over the (flat) coordinate and hence this case fits well into the general pattern. Then we consider $M(3 / 5)$ non-unitary model. Similarly to unitary $M(3 / 4)$ model, this model is based on two-dimensional Frobenius manifold. However, it turns out that the details of the construction are very different: compared to $M(3 / 4)$ case, where the cosmological constant and the parameter $x$ in the Douglas equation have the same gravitational scaling dimensions, the gravitational scaling dimension in $M(3 / 5)$ of these two parameters are different. This fact leads to an uncertainty in the choice of the integration contour in the flat coordinate space. Nevertheless, carefully employing the Douglas string equation together with the resonance transformations and with a conjectural choice of the contour allows to match this case with the FZZ Liouville results [39] as well. In section 5, we discuss some future perspectives. Some relevant formulae are listed in the appendix A.

\section{Dual approach to MLG}

In this section we summarize the basic elements of the dual approach to MLG and define a framework of computing 2D minimal gravity generating functions.

\subsection{Douglas equation and Frobenius manifolds}

The basic element of the matrix models approach (in the continuum limit) is $Q$ differential operator (for reviews, see $[52,53]$ ). In $A_{q-1}$ model it contains $q-1$ variables $u_{i}$ and is represented as

$$
Q=\partial^{q}+\sum_{i=1}^{q-1} u_{i}(x) \partial^{q-1-i} .
$$

The set $u=\left\{u_{i}\right\}$ is assumed to be a function of variable $x$, representing the continuum limit of the discrete state enumeration in the basis of orthogonal polynomials. ${ }^{3}$ The functional dependence of $u$ on $x$ at $p$-critical point is established by introducing $P=Q^{p / q}$ and requiring

$$
[P, Q]=1 .
$$

This constraint, so-called Douglas equation [12], in general reduces to higher order nonlinear differential equations.

On the other hand, the parameters $u_{i}$ can be considered as coordinates on a Frobenius manifold $\mathcal{M}$ with $\operatorname{dim}_{\mathcal{M}}=q-1$. The main property of $\mathcal{M}$ is the structure of a commutative and associative algebra with unity (to be specified below) defined in the tangent space at each point $u$ and compatible in a certain way [30] with the Riemannian structure of $\mathcal{M}$. By definition, Frobenius manifold is flat, so that there exist flat coordinates $v_{i}$, in which

\footnotetext{
${ }^{3}$ For simplicity, we omit here the dependence on the parameter responsible for the genus expansion. More detailed description can be found e.g. in the above mentioned references.
} 
the metric $\eta_{l k}$ on $\mathcal{M}$ is constant. For our purposes it will be convenient to use the flat basis and its tangent $e_{i}$. The multiplicative rule for the elements of the tangent basis is

$$
e_{i} e_{j}=c_{i j}^{k} e_{k},
$$

where in our convention $e_{1}=1$, so that $c_{1 j}^{k}=\delta_{j}^{k}$. Using $\eta_{l k}$ one may perform raising and lowering indices, e.g. $c_{i j k}=c_{i j}^{l} \eta_{l k}$. Defining property of $\mathcal{M}$ is that the structure constant $c_{i j k}$ is fully symmetric in the index permutation and obeys the following constraint

$$
\partial_{\ell} c_{i j k}=\partial_{k} c_{i j \ell}
$$

or, equivalently, $c_{i j k}=\partial_{i} \partial_{j} \partial_{k} F$, where $F$ is the so-called prepotential.

The flat coordinates $v$ can be constructed in terms of the coordinates $u$ using the following explicit form of the flat metric (see, e.g. [25, 27])

$$
\eta_{i j}=-q \operatorname{Res}_{y=\infty} \frac{e_{i} e_{j}}{Q^{\prime}(y, u)}=\delta_{i+j, q} .
$$

Here polynomial $Q(y, u)=y^{q}+\sum_{i=1}^{q-1} u_{i} y^{q-1-i}(\partial$ is replaced by a commuting number $y)$, $e_{i}=\partial Q(y, u) / \partial v^{i}$ and $Q^{\prime}(y, u)=\partial Q(y, u) / \partial y$. It gives the following expression for the flat coordinates $v_{i}=\theta_{i, 0}=\eta_{i j} v^{j}$, where ${ }^{4}$

$$
\theta_{i, k}=-\frac{\Gamma(i / q)}{\Gamma(i / q+k+1)} \operatorname{Res}_{y=\infty} Q^{k+i / q}(y),
$$

with non-negative integers $k$ and $1 \leq i \leq q-1$. The structure constants are also given explicitly

$$
c_{i j k}:=-q \operatorname{Res}_{y=\infty} \frac{e_{i} e_{j} e_{k}}{Q^{\prime}(y, u)}=\eta_{k l} c_{i j}^{l} .
$$

We note that $c_{1 i j}=\eta_{i j}$ since $e_{1}=1$.

\subsection{Scaling dimensions and $2 \mathrm{D}$ gravity}

Conformal field theoretical approach to $2 \mathrm{D}$ gravity emerged from the path integral formulation [1]. Liouville gravity is constructed as a direct product of three CFT's:

$$
\text { matter } \mathrm{CFT} \times \mathrm{LFT} \times(b, c) \text { ghosts },
$$

with central charges $c_{M}, c_{L}$ and $c_{g h}=-26$ respectively. The consistency of the theory requires the total central charge to vanish

$$
c_{L}+c_{M}+c_{g h}=0 .
$$

In fact, this condition guarantees that the theory admits a nilpotent BRST operator.

In the minimal Liouville gravity the matter sector is represented by $M(q / p)$ minimal model (with $q$ and $p$ coprime, $q<p$ ). The Liouville central charge $c_{L}=1+6 Q_{L}^{2}$, where the background charge $Q_{L}=b+1 / b$ and $b$ is the Liouville coupling constant, the minimal

\footnotetext{
${ }^{4}$ We introduce here more general object $\theta_{i, k}$, which will be useful in further consideration.
} 
model cenrtral charge $c_{M}$ is conveniently parametrized as $c_{M}=1-6 q_{M}^{2}$ with $q_{M}=1 / \beta-\beta$ and $\beta=\sqrt{q / p}$ or explicitly $c_{M}=1-6(p-q)^{2} / q p$. The total central charge balance condition (2.9) then requires $b=\beta$.

In the minimal Liouville gravity the field content of the matter minimal model is coupled to the Liouville vertex operators in the gravitational sector, such that the resulting "dressed" operator is BRST invarant. We consider the following BRST-invariant operators

$$
\mathbb{W}_{m, n}=c \cdot \bar{c} \cdot \mathbb{U}_{m, n}, \quad \widetilde{\mathbb{W}}_{m, n}=\int \mathrm{d}^{2} z \mathbb{U}_{m, n},
$$

where $\mathbb{U}_{m, n}=\Phi_{m, n} \cdot V_{a_{L}}$ is constructed from the primary fields $\Phi_{m, n}$ and $V_{a_{L}}$ in the matter and Liouville sectors respectively and $c(\bar{c})$ is the $(-1)$-weight ghost field. The conformal dimension of $\Phi_{m, n}=\Phi_{q-m, p-n}(1 \leq m<q, 1 \leq n<p)$ is $\Delta_{m, n}=\alpha_{m, n}\left(q_{M}+\alpha_{m, n}\right)$, where

$$
\alpha_{m, n}=\frac{|m / \beta-n \beta|-q_{M}}{2}=\frac{|m p-n q|-(p-q)}{2 p \beta}
$$

and the Liouville primary field $V_{a_{L}}$ has $\Delta_{a_{L}}^{L}=a_{L}\left(Q_{L}-a_{L}\right)$. The total conformal dimension vanishes $\Delta\left(\mathbb{W}_{m, n}\right)=\Delta\left(\widetilde{\mathbb{W}}_{m, n}\right)=0$, as a consequence of the BRST symmetry, leading to the constraint

$$
a_{L}=\alpha_{m, n}+\beta^{-1} .
$$

The conformal property of the Liouville gravity induces the gravitation scaling property, defining the reaction of the theory on the re-scaling of the (bulk) cosmological constant $\mu$. The partition function $Z_{L}$ on the sphere has the following scaling behavior [11]

$$
Z_{L} \sim \mu^{Q_{L} / b}
$$

so that its scaling dimension (g-dim) is $Q_{L} / b=(p+q) / q$, while the scaling dimensions of the physical fields (2.10) are equal to $a_{L} / b$. One can assign g-dim to the coupling constant $\mathcal{R}_{m n}$ accompanying fields in the MLG correlation numbers generating function $Z_{L}(\mathcal{R})=\left\langle\exp \sum_{m, n} \mathcal{R}_{m n} \mathbb{W}_{m, n}\right\rangle$, where $\mathcal{R}=\left\{\mathcal{R}_{m n}\right\}$ :

$$
\left[\mathcal{R}_{m n}\right]=\frac{p+q-|m p-n q|}{2 q}
$$

so that $(p+q) / q=\left[\mathcal{R}_{m n}\right]+\alpha_{m, n} / \beta+\beta^{-2}$. Here we use $[x]$ to denote the gravitation dimension (g-dim) of $x$.

The scaling properties play a key role in relating the observables of the matrix models with those of the Liouville gravity. For example, in the spherical topology the Douglas equation (2.2) is conveniently formulated using the action principle [54] $\partial S(u) / \partial u_{i}=0$ for the so-called Douglas action

$$
S(u)=\operatorname{Res}_{y=\infty}\left(Q^{\frac{p+q}{q}}+\sum_{m, n} t^{(m n)} Q^{\frac{|p m-q n|}{q}}\right) .
$$

Here the summation over $m$ and $n$ is restricted to the region $1 \leq m<q, 1 \leq n<p$ modulo equivalence $t^{(m n)}=t^{(q-m, p-n)}$. If one assigns g-dim $[Q]=1 / 2$, then taking into account 
that each term in (2.15) has the same g-dim one gets $\left[t^{(m n)}\right]=\left[\mathcal{R}_{m n}\right]$ defined in (2.14). This allows to relate the coupling constants $\mathcal{R}_{m n}$ of the Liouville gravity with the parameters $t^{(m n)}$ of the matrix models, up to some resonance terms to be discussed shortly.

The susceptibility condition imposes

$$
\frac{\partial^{2}}{\partial x^{2}} \mathcal{F}=v_{1}^{*}
$$

where $\mathcal{F}$ is the free energy of the matrix model and $v_{1}^{*}$ is a specific solution to the Douglas equation. Since $\mathcal{F}$ has the same g-dim as the Liouville partition function $Z_{L}, x$ becomes the parameter of the highest g-dim which is equal to $(p+q-1) / q ;[x]=\left[t^{\left(m_{1}, n_{1}\right)}\right]$ with $m_{1}, n_{1}$ subject to the constraint $\left|p m_{1}-q n_{1}\right|=1$.

The free energy $\mathcal{F}(t)$ has contributions from all genus partition functions. To get a particular genus part one may introduce a formal genus parameter both in the free energy and in the Douglas string equation (leading to the genus expansion of its solution) and to combine then relevant terms. For the genus 0 case, according to this procedure, we should replace $\partial$ in $Q$ in (2.15) by a commuting coordinate $y$. Using the notation $\theta_{i, k}$ introduced in $(2.6)$ one can write the action $S(u)$ in the form

$$
S(v, t)=\sum_{i=1}^{q-1} t_{i} v_{i}-H(v), \quad H(v, t)=\theta_{p_{0}, s+1}-\sum_{k \neq 0} t_{i, k} \theta_{i, k},
$$

where $p=s q+p_{0}$ with a non-negative integer $s$. The terms $\theta_{i, k \neq 0}$ are collected in $H(v)$. We note that the indices $i, k$ and $m, n$ are related as $i=|p m-q n|(\bmod q)$ and $k=(|p m-q n|-$ $i) / q \neq 0$. In what follows, in spite of this one-to-one correspondence, we keep sometimes (for convenience) double labeling $t_{i, k}^{(m n)}$ instead of $t_{i, k}$. We note that $\left[t_{i}^{\left(m_{i} n_{i}\right)}\right]=\left[\mathcal{R}_{m_{i} n_{i}}\right]$ and $\left[t_{i, k}^{(m n)}\right]=\left[\mathcal{R}_{m n}\right]$.

Even though g-dim of $t^{(m n)}$ and $\mathcal{R}_{m n}$ are the same, one cannot identify these two quantities. There are two reasons which prohibit such simple identification: the different normalization of the two approaches and the appearance of the resonances between operators, emerging from contact term interactions and not violating the scaling property

$$
t^{(m, n)}=\mathcal{R}_{m, n}+\sum A_{\left(m_{1}, n_{1}\right),\left(m_{2}, n_{2}\right)}^{(m, n)} \mathcal{R}_{m_{1}, n_{1}} \mathcal{R}_{m_{2}, n_{2}}+\cdots
$$

Here $A_{\left(m_{1}, n_{1}\right),\left(m_{2}, n_{2}\right)}^{(m, n)}$ are dimensionless constants and the sum goes over all possible combinations respecting the scaling. Since powers of $\mathcal{R}_{11} \propto \mu$ provide scaling dimensions, it is convenient to reformulate the resonance transformation as follows

$$
\begin{aligned}
t^{(m n)}= & \mathcal{R}_{m n}+A_{m n} \mu^{\delta_{m n}}+\sum_{m_{1}, n_{1}}^{\delta_{m_{1} n_{1}} \leqslant \delta_{m n}} A_{m n}^{m_{1} n_{1}} \mu^{\delta_{m n}-\delta_{m_{1} n_{1}}} \mathcal{R}_{m_{1} n_{1}} \\
& \delta_{m_{1} n_{1}+\delta_{m_{2} n_{2}} \leqslant \delta_{m n}} A_{m n}^{m_{1} n_{1}, m_{2} n_{2}} \mu^{\delta_{m n}-\delta_{m_{1} n_{1}}-\delta_{m_{2} n_{2}}} \mathcal{R}_{m_{1} n_{1}} \mathcal{R}_{m_{2} n_{2}}+\cdots, \\
& +\sum_{m_{1}, n_{1}, m_{2}, n_{2}}+\cdots
\end{aligned}
$$

where the non-vanishing coefficient can appear when the power of $\mu$ is a non-negative integer reflecting the possibility to perform a finite renormalization. 
There are different solutions to the Douglas equation $\partial S(v) /\left.\partial v_{i}\right|_{0^{*}}=0$, where the condition $0^{*}$ stands for the case, where all the couplings $\mathcal{R}_{m n}$ vanish except $\mathcal{R}_{11}$. It is known [27] that the minimal gravity is described by one particular solution such that $\left.v_{i>1}\right|_{0^{*}}=0$. In what follows, we call this on-shell solution and denote it $v^{*}$. Thus $v_{i>1}^{*}=0$ and the value $v_{1}^{*}$ is determined from the Douglas equation.

On the other hand, $\partial S / \partial v_{1}=0$ is the integrated form of (2.2), whose integration variable is $x$. Since $x$ and $t_{1}$ have the same g-dim, two variables are closely related. For later convenience, we define $x$

$$
x:=\frac{\partial H(v)}{\partial v_{1}},
$$

which gives implicit relation between $v_{i}$ 's and $x$. If one takes the derivative with respect to $x$, eq. (2.20) reduces to eq. (2.2). Since $\partial S(v) / \partial v_{1}=t_{1}-x$, we have $x=\partial H(v) /\left.\partial v_{1}\right|_{0^{*}}=$ $\left.t_{1}\right|_{0^{*}}$ as the one-shell solution. The distinction of $x$ from $t_{1}$ is significant because the functional relation of $v$ with $x$ holds even off-shell, where $t_{1}$ (considered as coupling constant) is to be identified with $\mathcal{R}_{m_{1} n_{1}}$.

The free energy $\mathcal{F}$ has to satisfy the susceptibility condition (2.16). From this property, the free energy is [26]

$$
\mathcal{F}(t)=\frac{1}{2} \int_{0}^{v^{*}} c_{\beta \gamma}^{\alpha} \frac{\partial S}{\partial v_{\beta}} \frac{\partial S}{\partial v_{\gamma}} \mathrm{d} v_{\alpha} .
$$

Because the integrand is the closed one-form [25], taking into account the property of the relevant solution $v^{*}$, it is convenient to chooses the integration path $d v_{i>0}=0$, then the free energy has simpler form

$$
\mathcal{F}(t)=\frac{1}{2} \int_{0}^{v_{1}^{*}} c_{\beta \gamma}^{1} \frac{\partial S}{\partial v_{\beta}} \frac{\partial S}{\partial v_{\gamma}} \mathrm{d} v_{1} .
$$

Bulk correlation numbers are obtained by differentiating with respect to the corresponding coupling constants. Thus, (2.22) is the generating function of the bulk correlation on the sphere.

\subsection{Bulk generating function on the disk}

The bulk free energy on the disk is obtained using the free energy idea of one matrix model. It is shown in [46] that the boundary free energy with $h \geq 1$ holes (boundaries) is given by

$$
\mathcal{F}_{h}(t)=\frac{1}{h !}\left\langle(-\operatorname{Tr} \log C(M))^{h}\right\rangle_{c}=\frac{1}{h !}\left\langle\left(\int_{0}^{\infty} \frac{d l}{l} \operatorname{Tr} e^{-l C(M)}\right)^{h}\right\rangle_{c},
$$

where the expectation value is evaluated with respect to the bulk interaction and the subscript $c$ refers to the connected part. Tr acts on the matrix components. With the one boundary loop $(\mathrm{h}=1)$ one has the free energy on the disk.

$$
\mathcal{F}_{1}(t)=-\left\langle\operatorname{Tr} \log \left(\mu_{B}-M\right)\right\rangle_{c}=\int_{0}^{\infty} \frac{d l}{l} e^{-\ell \mu_{B}} \operatorname{Tr}\left\langle e^{l M}\right\rangle
$$

Here we use the prescription $C(M)=\mu_{B}-M$ which describes the case with no boundary operators with $\mu_{B}$ boundary cosmological constant. The derivative of $\mathcal{F}_{1}$ with respect to 
$\mu_{B}$ is the one-point resolvent expectation value. It is noted that the integration over $l$ is the Laplace transform, and the integration path is chosen form 0 to $\infty$ assuming the contribution due to $\operatorname{Tr}\left\langle e^{l M}\right\rangle$ is convergent. If not, the integration range is properly redefined so that the integration converged.

The expectation value $\operatorname{Tr}\left\langle e^{l M}\right\rangle$ is the main element for finding the free energy on the disk. At the continuum limit, $M$ is replaced by $Q$. In [21] the element is treated as the expectation value of the loop operator $\langle w(l)\rangle$. We will denote the expectation value as $\mathcal{W}(l)$.

$$
\mathcal{W}(l)=\int_{x_{1}}^{\infty} \mathrm{d} x\left\langle x\left|e^{l Q}\right| x\right\rangle=\int_{x_{1}}^{\infty} \mathrm{d} x \int_{i \mathbb{R}} \mathrm{d} y e^{l Q(y, v(x))},
$$

where $Q$ is given in $x$-representation. Tr becomes the integration over $x$ and the integration interval $x_{1}$ is to be identified with $t^{\left(m_{1}, n_{1}\right)}$.

The generating function on the disk with genus 0 is obtained if $\partial$ is replaced by $y$ in $Q$. Since $y$ represents the Fourier space, we have put $Q=Q(y, v(x))$ and integration of $y$ is performed over $i \mathbb{R}$. In addition, the flat coordinates $v$ have $x$-dependence through (2.20), which is different from on-shell value $v^{*}$. On the sphere the bulk generating function does not contain explicit $x$-dependence of $v$. However, on the disk explicit $x$-dependence is necessary. On the disk we need $Q(y, v(x, t))$ and more information about $x$ and coupling constant dependence is required in order to get the generating function $\mathcal{W}(l, t)$.

The bulk correlation on the disk is given as a certain derivative of the bulk generating function (2.25). For example, one-point correlation is given as

$$
\left\langle O_{m, n}\right\rangle_{D}=\left.\frac{\partial}{\partial \mathcal{R}_{m, n}} \mathcal{W}(l)\right|_{0 *},
$$

where $0^{*}$ has the same meaning as in the spherical case. The result (2.26) is to be compared with FZZ result in [39]

$$
W_{\alpha_{L}}\left(l_{0}\right)=\frac{2}{b}\left(\pi \mu \gamma\left(b^{2}\right)\right)^{\left(Q_{L}-2 \alpha\right) / 2 b} \frac{\Gamma\left(2 \alpha b-b^{2}\right)}{\Gamma\left(1+1 / b^{2}-2 \alpha / b\right)} K_{\left(Q_{L}-2 \alpha_{L}\right) / b}\left(\kappa l_{0}\right),
$$

where $\kappa^{2}=\mu / \sin \left(\pi b^{2}\right)$ and the order of the Bessel function is given as $\nu_{m, n}=\frac{Q_{L}-2 \alpha_{m, n}}{b}=$ $\frac{|m p-n q|}{q}$. More specifically for the minimal model, FZZ result has the form

$$
W_{\alpha_{L}}\left(l_{0}\right)=e_{\alpha_{L}} \kappa^{\nu_{m, n}} K_{\nu_{m, n}}\left(\kappa l_{0}\right)
$$

where $e_{\alpha_{L}}$ is a certain numerical constant independent of $\kappa$ and $l_{0}{ }^{5}$ and is related with the normalization of the coupling parameters as in the resonance transformations (2.18). Non-trivial task is to compare the result of one-point correlation on disk of two different approaches and check if the argument $\nu_{m, n}$ of the Bessel function matches each other. Explicit checks aimed at testing (2.26) against (2.28) will be the subject of the next two sections.

\footnotetext{
${ }^{5}$ We note that the length parameters $l$ and $l_{0}$ in $(2.25)$ and (2.27) are different. The relation between them is model dependent and will be fixed in our concrete examples. This is because there is no canonical normalization.
} 
We note that formula (2.24) gives correlation with the simplest boundary condition (corresponding to $(1,1)$ Cardy boundary condition). More complicated boundary conditions (known as $(k, l)$ Cardy branes) can be obtained by modifying (2.24) but still maintaining the formula (2.25). This is because other non-trivial boundary conditions are obtained as the superposition of FZZ branes, replacing the boundary parameter $\mu_{B}$ in (2.24) with the set of complex boundary parameters $\cosh \left(\pi s_{m, n} / p\right)$ with $s_{m, n}=s+i m q+i n p$ where $s$ is a real parameter, integer $m$ runs from $-(l-1)$ to $(l-1)$ in two steps and $n$ from $-(k-1)$ to $(k-1)$ in two steps as suggested in $[46,48,51,55]$. This additional non-trivial boundary conditions will be discussed in a separate paper.

\section{$3 \quad$ Unitary models}

In this section we compute one-point functions for the unitary series of Minimal Gravity in the "dual" aproach using the existing results on the resonance transformation. We compare our results with the ones from the Liouville gravity.

\subsection{M(3/4): Ising model — the simplest unitary case}

According to the general framework discussed above, the Ising model is governed by the $A_{2}$ Frobenius manifold with the Lax polynomial $Q=y^{3}+v_{1} y+v_{2}$, where $v_{1}$ and $v_{2}$ are the flat coordinates. The structure constants are $c^{122}=1, c^{111}=-v_{1} / 3$ and the nontrivial component of the metric is $\eta_{12}=1$. The Douglas action has the form

$$
\begin{aligned}
S & =t_{1}^{(11)} v_{1}+t_{2}^{(12)} v_{2}-H, & H & =\theta_{1,2}-t_{2,1}^{(13)} \theta_{21}, \\
\theta_{1,2} & =\left(-v_{1}^{4}+18 v_{1} v_{2}^{2}\right) / 36, & \theta_{2,1} & =\left(-v_{1}^{3}+9 v_{2}^{2}\right) / 18,
\end{aligned}
$$

where $(m, n)$ index has the $Z_{2}$ symmetry: $t_{1}^{(11)}=t_{1}^{(23)}, t_{2}^{(12)}=t_{2}^{(22)}, t_{2,1}^{(13)}=t_{2,1}^{(21)}$. The gravitational dimensions of the coupling constants are

$$
\left[t_{1}^{(11)}\right]=1, \quad\left[t_{2}^{(12)}\right]=5 / 6, \quad\left[t_{2,1}^{(13)}\right]=2 / 6 .
$$

Since $t_{1}^{(11)}$ has g-dim 1 the following resonance terms are allowed

$$
\begin{aligned}
t_{1}^{(11)} & =\mathcal{R}_{11}+A_{(21),(21),(21)}^{(11)}\left(\mathcal{R}_{13}\right)^{3}, \\
t_{2}^{(12)} & =\mathcal{R}_{12}, \\
t_{2,1}^{(13)} & =\mathcal{R}_{13} .
\end{aligned}
$$

The derivatives of $S$ are

$$
\begin{aligned}
& \frac{\partial S}{\partial v_{1}}=t^{(11)}-x, \quad \text { where } \quad x=-\frac{1}{9} v_{1}^{3}+\frac{1}{2} v_{2}^{2}+\frac{1}{6} t_{21}^{(13)} v_{1}^{2}, \\
& \frac{\partial S}{\partial v_{2}}=t_{2}^{(12)}+t_{21}^{(13)} v_{2}-v_{1} v_{2} .
\end{aligned}
$$

The Douglas equation reads

$$
\frac{\partial S}{\partial v_{1}}=\frac{\partial S}{\partial v_{2}}=0
$$


From (3.7), (3.8) it follows that the on-shell solution (all $t=0$ except $t^{(11)} \sim \mu$ ) is $v_{2}^{*}=$ $0,\left(v_{1}^{*}\right)^{3}=9 \mathcal{R}_{11}$. In addition, $x$-dependence of $v_{i}$ 's is fixed by the second equation in (3.7).

Bulk generating function on the sphere is [26]

$$
\mathcal{F}(t)=\frac{1}{2} \int^{v_{1}^{*}} d v_{1}\left(\left(\frac{\partial S}{\partial v_{1}}\right)^{2}-\frac{v_{1}}{3}\left(\frac{\partial S}{\partial v_{2}}\right)^{2}\right) .
$$

One can check that the bulk one-point correlation numbers on the sphere vanish. Note that $\left[O_{12}\right]=3 / 2$ and $\left\langle O_{12}\right\rangle$ is non-analytic in $\mu$ and this correlator must vanish to fulfil selection rules. Indeed, explicit evaluation shows that

$$
\left\langle O_{12}\right\rangle=\left.\frac{\partial \mathcal{F}(t)}{\partial \mathcal{R}_{12}}\right|_{0^{*}}=\int_{0}^{v_{1}^{*}} d v_{1}\left(-\frac{v_{1}}{3}\right)\left(-v_{1} v_{2}^{*}\right),
$$

which is identically zero due to the on-shell value $v_{2}^{*}=0$ deternined in (3.8). On the other hand, $\left[O_{13}\right]=2$ and

$$
\left\langle O_{13}\right\rangle=\left.\frac{\partial \mathcal{F}(t)}{\partial \mathcal{R}_{13}}\right|_{0^{*}}=\int_{0}^{v_{1}^{*}} d v_{1}\left(\mu+\frac{v_{1}^{3}}{9}\right)\left(-\frac{v_{1}^{2}}{6}\right) \propto \mu^{2} .
$$

The non-vanishing contribution is analytic in $\mu$ and is not universal, so that it can be discarded. This shows that $\left\langle O_{13}\right\rangle=0\left(\bmod \mu^{2}\right)$. One may also check that two-point correlator $\left\langle\mathrm{O}_{22} \mathrm{O}_{21}\right\rangle$ vanishes automatically since $v_{2}^{*}=0$.

Bulk one-point correlation on the disk is defined according to (2.26). Its generating function is $\mathcal{W}(l, t)=\int_{\mu}^{\infty} \mathrm{d} x\left\langle x\left|e^{l Q}\right| x\right\rangle$. The polynomial $Q\left(y, v_{1}\right)=y^{3}+v_{1} y$ can be written in terms of Chebyshev polynomial $T_{3}(x)=4 x^{3}-3 x$

$$
Q\left(y, v_{1}\right)=2 i\left(v_{1} / 3\right)^{3 / 2} T_{3}\left(\frac{i y \sqrt{3}}{2 \sqrt{v_{1}}}\right) .
$$

Therefore, we have

$$
\left\langle O_{11}\right\rangle_{D}=\int_{\mathbb{R}} \mathrm{d} y e^{i l\left(y^{3}+v_{1 *} y\right)}=\frac{2 v_{1 *}^{1 / 2}}{3} K_{1 / 3}\left(2 l\left(v_{1 *} / 3\right)^{3 / 2}\right),
$$

where on-shell value $v_{2 *}=0, v_{1 *}=(9 \mu)^{1 / 3}$ is used.

To evaluate other one-point functions we need to compute $\frac{\partial Q(y, v(x))}{\partial \mathcal{R}_{m, n}}$ on-shell. For this purpose we use the Douglas equation (3.7) and (3.8)

$$
\left.\frac{\partial v_{1}}{\partial t_{2,1}^{(21)}}\right|_{0^{*}}=-\frac{1}{2},\left.\quad \frac{\partial v_{2}}{\partial t_{2}^{(22)}}\right|_{0^{*}}=\frac{1}{v_{1}} .
$$

This shows that

$$
\left\langle O_{2,2}\right\rangle_{D}=l \int_{\mu}^{\infty} \frac{\mathrm{d} x}{v_{1}} \int_{\mathbb{R}} \mathrm{d} y e^{i l\left(y^{3}+v_{1} y\right)}=-\frac{4 l v_{1 *}}{3 z} K_{2 / 3}\left(z \mu^{1 / 2}\right), \quad z=2 l \sqrt{3},
$$


where we used the integration formulae from the appendix A. This coincides with the FZZ result (2.27). Similarly we have

$$
\left\langle O_{1,3}\right\rangle_{D}=-\frac{4}{9 z} i l v_{1}^{5 / 2} K_{5 / 3}\left(z \mu^{1 / 2}\right) .
$$

In particular we find the relation between $l$ and $l_{0}$ from (2.28): $l / l_{0}=2^{3 / 4} / \sqrt{3}$.

\section{$3.2 \mathrm{M}(\mathrm{q} / \mathrm{q}+1)$ : towards the general case}

Now we describe the general unitary minimal model $(q, p)=(q, q+1)$. Corresponding Frobenius manifold is $A_{q-1}$ and the Lax operator is $Q=y^{q}+\sum_{i=1}^{q-1} u_{i} y^{q-1-i}$. The flat coordinates of the $q$-1-dimensional Frobenius manifold are given as $v_{i}=\theta_{i, 0}=u_{i}+\cdots$ and the metric is $\eta_{i j}=\delta_{i+j, q}$.

In general the structure constants are complicated. However, the bulk correlation generating function needs the structure constants $c_{i j}^{1}$ on-shell only, which follows from the condition $v_{i>1}^{*}=0$. It was shown in [26] that $c_{i j}^{1}$ on-shell has non-vanishing components when $i=j$ only and is given explicitly as

$$
c_{i j}^{1}=\delta_{i j} c_{i} v_{1}^{i-1}
$$

$c_{i}$ being a constant.

We have the action $S(v)$ of the form

$$
S(v)=\sum_{i} v_{i} t_{i}^{(i, i)}-H(v), \quad H(v)=\theta_{1,2}(v)-\sum_{j=1}^{q-1} \sum_{k \geq 1} t_{j, k}^{(m, n)} \theta_{j, k}(v),
$$

where $(k=m-n, j=m)$ or $(m=j, n=j-k)$. The parameter $t$ has the symmetry $t_{i, k}^{(m, n)}=t_{i, k}^{(q-m, p-m)}$. The highest g-dim is 1 and therefore we have $[x]=\left[\mathcal{R}_{11}\right]$. The derivative of the action is $\partial S / \partial v_{i}=t_{i}^{(i, i)}-\partial H(v) / \partial v_{i}$ with

$$
\frac{\partial H(v)}{\partial v_{i}}=\frac{\partial \theta_{1,2}(v)}{\partial v_{i}}-\sum_{j=1}^{q-1} \sum_{k \geq 1} t_{j, k}^{(m, n)} \frac{\partial \theta_{j, k}(v)}{\partial v_{i}} .
$$

The Douglas equation $\partial S / \partial v_{i}=0$ has the on-shell solution $v_{i>1}^{*}=0$ and on-shell value of $Q$ is given in terms Chebyshev polynomial $T_{q}[56]$

$$
Q\left(y, v_{1}\right)=2(-i)^{q}\left(\frac{v_{1}^{*}}{q}\right)^{q / 2} T_{q}\left(i y \frac{q^{1 / 2}}{2 v_{1}^{* 1 / 2}}\right)=y^{n}+y^{n-2} v_{1}^{*}+\cdots .
$$

The derivative of the action for $\left(v_{i>0}=0\right)$ can be found explicitly using

$$
\begin{array}{ll}
\frac{\partial \theta_{j, k}(v)}{\partial v_{i}}=\delta_{j, i} x_{j, k}\left(-v_{1} / q\right)^{k q / 2} & \text { when } \quad k \text { even } \\
\frac{\partial \theta_{j, k}(v)}{\partial v_{i}}=\delta_{j, q-i} y_{j, k}\left(-v_{1} / q\right)^{(k-1) q / 2+j} & \text { when } \quad k \text { odd },
\end{array}
$$


where

$$
x_{j, k}=\frac{1}{\left(\frac{j}{m}\right)_{\frac{k}{2}}\left(\frac{k}{2}\right) !}, \quad y_{j, k}=-\frac{1}{\left(\frac{j}{m}\right)_{\frac{k-1}{2}}\left(\frac{k-1}{2}\right) !}
$$

and $(a)_{n}=\Gamma(a+n) / \Gamma(a)$ is a Pochhammer symbol. The bulk generating function on the sphere is

$$
\mathcal{F}(t)=\sum_{i=1}^{q-1} \frac{c_{i}}{2} \int_{0}^{v_{1}^{*}} d v_{1} v_{1}^{i-1}\left(\frac{\partial S}{\partial v_{i}}\right)^{2},
$$

where the integration path is along $v_{i>0}=0$. In addition, the structure constant $c_{j m}^{1}$ in (3.18) is used. Differentiating this function and requiring one-point function vanishing, diagonality of two-point function and other fusion rules, the resonance transformation were found in [26]. To this end, we introduce a new integration variable $w$

$$
w=2\left(\frac{v_{1}}{v_{1}^{*}}\right)^{q}-1
$$

The integration now looks like

$$
\int_{0}^{v_{1}^{*}} \frac{d v_{1}}{v_{1}}\left(\frac{v_{1}}{v_{1}^{*}}\right)^{q} \rightarrow \frac{1}{2 q} \int_{-1}^{1} d w
$$

In the new variable the fusion rules become the orthogonality conditions for Jacobi polynomials

$$
\frac{1}{q} \int_{-1}^{1} d w(1+w)^{\beta} P_{m}^{(0, \beta)} P_{n}^{(0, \beta)}=\delta_{m n} \frac{2^{\beta+1}}{2 n+\beta+1} .
$$

In particular, if $\beta=0$ Jacobi polynomial reduces to Legendre polynomial.

The generating function on the disk is given in (2.25) and its one-point correlation is given in (2.26). Explicitly one gets (if $(m, n) \neq(1,1))^{6}$

$$
\left\langle O_{m, n}\right\rangle_{D}=l \int_{\mu}^{\infty} \mathrm{d} x \int_{\mathbb{R}} \mathrm{d} y \frac{\partial Q(y, v)}{\partial \mathcal{R}_{m, n}} e^{l Q(y, v)} .
$$

Pre-exponent is evaluated using

$$
\frac{\partial Q(y)}{\partial v_{\alpha}} \frac{\partial v_{\alpha}}{\partial \mathcal{R}_{m, n}}=\frac{\partial Q(y)}{\partial u_{a}} \frac{\partial u_{a}}{\partial v_{\alpha}} \frac{\partial v_{\alpha}}{\partial \mathcal{R}_{m, n}} .
$$

We use the expressions for the second and third terms from the paper [26]

$$
\begin{gathered}
\partial v_{\alpha} / \partial \mathcal{R}_{m, n}=-\partial_{v_{\beta}} S^{(m, n)}\left[\left(\frac{\partial^{2} S_{0}}{\partial v^{2}}\right)^{-1}\right]^{\alpha, \beta}, \\
{\left[\left(\frac{\partial^{2} S_{0}}{\partial v^{2}}\right)^{-1}\right]^{\alpha, \beta}=\frac{1}{v_{1}}\left(\frac{-v_{1}}{q}\right)^{1-q+\alpha} \delta^{\alpha \beta} .}
\end{gathered}
$$

\footnotetext{
${ }^{6}$ In the exponent the sign should be picked in a way that the integral converges. That is $-l Q(y, v)$ if $q$ is even and $i l Q(y, l)$ if $q$ is odd.
} 
Here $S^{(m, n)}:=\partial S / \partial t_{i, j}^{(m, n)}$ and their derivatives $\partial S^{(m, n)} / \partial v_{a}$ are given in terms of the Jacobi polynomials $[26]$

$$
\begin{aligned}
& \left.\frac{\partial S^{(m, n)}}{\partial v_{a}}\right|_{0^{*}}=-\delta_{a, m}\left(\frac{m}{q}\right)_{m-n}^{-1}\left(\frac{-v_{1}^{*}}{q}\right)^{\frac{m-n}{2} q} P_{\frac{m-n}{2}}^{\left(0, \frac{m}{q}-1\right)}(w), \\
& \left.\frac{\partial S^{(m, n)}}{\partial v_{a}}\right|_{0^{*}}=\delta_{q-a, m}\left(\frac{m}{q}\right)_{m-n}^{-1}\left(\frac{-v_{1}^{*}}{q}\right)^{\frac{m-n-1}{2} q}\left(\frac{-v_{1}}{q}\right)^{m} P_{\frac{m-n-1}{2}}^{\left(0, \frac{m}{q}\right)}(w) .
\end{aligned}
$$

From these expressions it is clear that differentiating w.r.t. $\mathcal{R}_{m n}, m>n$ changes only $v_{m}$ if $m-n$ is even and $v_{q-m}$ otherwise. Using the formulae for $\partial u_{a} / \partial v_{b}$ from [26] we evaluate

$$
\frac{\partial Q(y, v)}{\partial v_{q-\gamma}}=(-i)^{\gamma-1}\left(\frac{v_{1}}{q}\right)^{\frac{\gamma-1}{2}} T_{\gamma}^{\prime}\left(i y \frac{\sqrt{q}}{2 \sqrt{v_{1}}}\right) .
$$

Thus, using expression (3.28) and formulae above we obtain

$$
\begin{array}{ll}
\left\langle O_{m, n}\right\rangle_{D} \sim l \int_{\mu} \mathrm{d} x \int_{\mathbb{R}} \mathrm{d} \tilde{y} v_{1}^{\frac{m-q}{2}} v_{1}^{* \frac{m-n}{2} q} P_{\frac{m-n}{2}}^{\left(0, \frac{m}{q}-1\right)}(w) T_{q-m}^{\prime}(\tilde{y}) \exp \left[-z T_{q}(\tilde{y})\right], & (m-n) \text { even }, \\
\left\langle O_{m, n}\right\rangle_{D} \sim l \int_{\mu} \mathrm{d} x \int_{\mathbb{R}} \mathrm{d} \tilde{y} v_{1}^{\frac{m}{2}} v_{1}^{* \frac{m-n-1}{2} q} P_{\frac{m-n-1}{2}}^{\left(0, \frac{m}{q}\right)}(w) T_{m}^{\prime}(\tilde{y}) \exp \left[-z T_{q}(\tilde{y})\right], & (m-n) \text { odd },
\end{array}
$$

where $z=2\left(v_{1} / q\right)^{q / 2} l$ and $\tilde{y}=\operatorname{sh} s=y \sqrt{q} /\left(2 \sqrt{v_{1}}\right)$. Using formulae from the appendix A if $q$ is even we get

$$
\int_{\mathbb{R}} \mathrm{d} y \partial Q(y, v) / \partial \mathcal{R}_{m, n} e^{-l Q(y, v)}=\begin{array}{|l|c|r|}
\hline & m-n \text { even } & m-n \text { odd } \\
\hline m \text { even } & 0 & 0 \\
\hline m \text { odd } & -C_{1}\left[v_{1}, v_{1}^{*}\right] K_{1-\frac{m}{q}}(z) & C_{2}\left[v_{1}, v_{1}^{*}\right] K_{\frac{m}{q}}(z) \\
\hline
\end{array}
$$

where

$$
\begin{aligned}
C_{1}\left[v_{1}, v_{1}^{*}\right] & =4 \frac{q-m}{q^{2}}\left(\frac{m}{q}\right)_{m-n}^{-1}\left(\frac{v_{1}}{q}\right)^{\frac{m-q}{2}}\left(\frac{v_{1}^{*}}{q}\right)^{\frac{m-n}{2} q} P_{\frac{m-n}{2}}^{\left(0, \frac{m}{q}-1\right)}(w) \\
& =\text { const. } v_{1}^{\frac{(m-n-1) q+m}{2}}+\text { const. } v_{1}^{\frac{(m-n-3) q+m}{2}} v_{1}^{* q}+\cdots \\
C_{2}\left[v_{1}, v_{1}^{*}\right] & =4 \frac{m}{q^{2}}\left(\frac{m}{q}\right)_{m-n}^{-1}\left(\frac{v_{1}}{q}\right)^{\frac{m}{2}}\left(\frac{v_{1}^{*}}{q}\right)^{\frac{m-n-1}{2} q} P_{\frac{m-n-1}{2}}^{\left(0, \frac{m}{q}\right)}(w) \\
& =\text { const. } v_{1}^{\frac{(m-n-1) q+m}{2}}+\text { const. } v_{1}^{\frac{(m-n-3) q+m}{2}} v_{1}^{* q}+\cdots
\end{aligned}
$$

and if $q$ is odd

$$
\int_{\mathbb{R}} \mathrm{d} y \partial Q(y, v) / \partial \mathcal{R}_{m, n} e^{i l Q(y, v)}=
$$

\begin{tabular}{|l|c|r|}
\hline & $m-n$ even & $m-n$ odd \\
\hline$m$ even & $-(-1)^{\frac{m-n}{2}} \cos \left(\frac{\pi}{2 q}\right) C_{1}\left[v_{1}, v_{1}^{*}\right] K_{1-\frac{m}{q}}(z)$ & $-i(-1)^{\frac{m-n-1}{2}} \sin \left(\frac{\pi}{2 q}\right) C_{2}\left[v_{1}, v_{1}^{*}\right] K_{\frac{m}{q}}(z)$ \\
\hline$m$ odd & $i(-1)^{\frac{m-n}{2}} \sin \left(\frac{\pi}{2 q}\right) C_{1}\left[v_{1}, v_{1}^{*}\right] K_{1-\frac{m}{q}}(z)$ & $-(-1)^{\frac{m-n-1}{2}} \cos \left(\frac{\pi}{2 q}\right) C_{2}\left[v_{1}, v_{1}^{*}\right] K_{\frac{m}{q}}(z)$ \\
\hline
\end{tabular}


To get one-point functions we need to integrate $\int_{\mu} \mathrm{d} x$ the results of (3.34) and (3.36). At the moment we are not aware how to perform it in the general case, however in all concrete examples we get

$$
\begin{aligned}
\left\langle O_{m, n}\right\rangle_{D} & \sim \mu^{\nu / 2} K_{\nu}\left(l \mu^{1 / 2}\right), & \nu & =m-n+\frac{m}{q}, \\
\left\langle O_{m, n}\right\rangle_{D} & \sim 0, & m, q & \in 2 \mathbb{Z} .
\end{aligned}
$$

At first sight it appears that the second case is in contradiction with the FZZ result. One of the reasons may be the symmetric minimal model boundary conditions chosen by matrix model integrals, however to check this we need to fix exact normalizations between MLG and Douglas equation approach. This phenomenon resembles also certain nonvanishing correlators appearing in the spherical case, which should vanish in the CFT approach (for the discussion see e.g. $[25,26])$.

For $m=n$ and $m=n+1$, Jacobi polynomial reduces to a constant and the integrals can be taken for all $\mathrm{q}$ and $\mathrm{m}$

$$
C_{1}\left[v_{1}, v_{1}^{*}\right]=4 \frac{q-m}{q^{2}}\left(\frac{v_{1}}{q}\right)^{\frac{m-q}{2}}, \quad C_{2}\left[v_{1}, v_{1}^{*}\right]=4 \frac{m}{q^{2}}\left(\frac{v_{1}}{q}\right)^{\frac{m}{2}} .
$$

Therefore we get if $\mathrm{q}$ is even, then $\mathrm{m}$ is odd and

$$
\left\langle O_{m, n}\right\rangle_{D}=\begin{array}{|c|c|r|}
\hline & m=n & m=n+1 \\
\hline m \text { odd } & -4 \frac{q-m}{\tilde{z} q^{2}}\left(\frac{v_{1}^{*}}{q}\right)^{\frac{m}{2}} K_{\frac{m}{q}}\left(z_{*}\right) & 4 \frac{m}{\tilde{z} q^{2}}\left(\frac{v_{1}^{*}}{q}\right)^{\frac{m+q}{2}} K_{1+\frac{m}{q}}\left(z_{*}\right) \\
\hline
\end{array}
$$

where $z_{*}=\left.z\right|_{v_{1}=v_{1}^{*}}$ and $\tilde{z}=z_{*} /(2 l \sqrt{\mu})=\sqrt{\frac{q}{(q+1)(2 q+1)}}$. Similarly for odd $q$ we obtain

\begin{tabular}{|l|c|r|}
\hline & $m=n$ & $m=n+1$ \\
\cline { 2 - 3 } \multicolumn{1}{|c|}{$m$ even } & $-4 \cos \left(\frac{\pi}{2 q}\right) \frac{q-m}{\tilde{z}_{*} q^{2}}\left(\frac{v_{1}^{*}}{q}\right)^{\frac{m}{2}} K_{\frac{m}{q}}\left(z_{*}\right)$ & $-4 i \sin \left(\frac{\pi}{2 q}\right) \frac{m}{\tilde{z}_{*} q^{2}}\left(\frac{v_{1}^{*}}{q}\right)^{\frac{m+q}{2}} K_{1+\frac{m}{q}}\left(z_{*}\right)$ \\
\hline$m$ odd & $4 i \sin \left(\frac{\pi}{2 q}\right) \frac{q-m}{\tilde{z}_{*} q^{2}}\left(\frac{v_{1}}{q}\right)^{\frac{m}{2}} K_{\frac{m}{q}}\left(z_{*}\right)$ & $-4 \cos \left(\frac{\pi}{2 q}\right) \frac{m}{\tilde{z}_{*} q^{2}}\left(\frac{v_{1}^{*}}{q}\right)^{\frac{m+q}{2}} K_{1+\frac{m}{q}}\left(z_{*}\right)$ \\
\hline
\end{tabular}

We also write down the partition function

$$
Z_{B}=2 c \frac{v_{1}^{* \frac{q+1}{2}}}{l \tilde{z}_{*} q^{3 / 2}} K_{1+\frac{1}{q}}\left(z_{*}\right),
$$

where $c=1$ for even q and $c=\cos (\pi / 2 q)$ for odd $\mathrm{q}$.

\section{Non-unitary models}

\section{1 $M(2 / 2 s+1):$ Lee-Yang series}

We revisit first the Lee-Yang series on the disk in [49] and discuss then the normalization effect. The Lee-Yang series $M(2 / p)$ with $p=2 s+1$ is described by one-matrix model 
and is based on $A_{1}$ Frobenius manifold with $Q=y^{2}+v$. The metric is trivially given by $\eta_{1,1}=1$. Nevertheless, the Douglas action is not trivial

$$
S(v)=t_{1}^{(1, s)} v-H(v), \quad H(v)=\frac{1}{s+2} v^{s+2}-\sum_{k=1}^{s-1} \frac{t_{1, k}^{\left(1, n_{k}\right)}}{k+1} v^{k+1}
$$

where $n_{k}=s-k \geq 1$. We use the fact that $\theta_{1, k}(v) \sim v^{k+1}$ and put some normalization constant for later convenience. The parameter $t$ has $\mathbb{Z}_{2}$ symmetry $t_{1, k}^{\left(1, n_{k}\right)}=t_{1, k}^{\left(1, p-n_{k}\right)}$ and g-dim of $t$ is $\left[t^{(1, n)}\right]=(1+n) / 2$. The highest g-dim is $(1+s) / 2$ and therefore, $\left[t_{1}^{(1, s)}\right]=$ $[x]=(1+s) / 2$, whereas $\left[t_{1, s-1}^{(1,1)}\right]=\left[\mathcal{R}_{11}\right]=1$. We remind that $\mathcal{R}_{1,1}=\mu$. Considering g-dim, the resonance transformation is as follows. When $n$ is odd $(\neq 1)$ and $\left[t^{(1, n)}\right]$ is integer

$$
\begin{aligned}
t^{(1, n)}=\mathcal{R}_{1, n} & +A_{(1, n)}^{(1,1)^{(n+1) / 2}} \mu^{(n+1) / 2}+\sum_{a=3,5,7 \cdots} A_{(1, n)}^{(1,1)^{(n-a) / 2},(1, a)} \mu^{(n-a) / 2} \mathcal{R}_{1, a} \\
& +\sum_{a+b=3,5,7 \cdots} A_{(1, n)}^{(1,1)^{(n-a-b-1) / 2},(1, a)(1, b)} \mu^{(n-a-b-1) / 2} \mathcal{R}_{1, a} \mathcal{R}_{1, b}+O\left(\mathcal{R}^{3}\right) .
\end{aligned}
$$

When $n$ is even and $\left[t^{(1, n)}\right]$ is half odd integer

$$
\begin{aligned}
t_{1, k}^{(1, n)}=\mathcal{R}_{1, n} & +\sum_{a=3,5,7 \cdots} A_{(1, n)}^{(1,1)^{n / 2-a},(1, a)} \mu^{n / 2-a} \mathcal{R}_{1,2 a} \\
& +\sum_{a+b=o d d} A_{(1, n)}^{(1,1)^{(n-a-b-1) / 2},(1, a)(1, b)} \mathcal{R}_{1,1}^{(n-a-b-1) / 2} \mathcal{R}_{1, a} \mathcal{R}_{1, b}+O\left(\mathcal{R}^{3}\right)
\end{aligned}
$$

The derivative of the action is $\partial S / \partial v \equiv t^{(1, s)}-x$, where

$$
x:=\frac{\partial H(v)}{\partial v}=v^{s+1}-t^{(1,1)} v^{s-1}-\sum_{k=1}^{s-2} t^{(1, s-k)} v^{k},
$$

which presents the implicit dependence of $v$ on $x$. Using the resonance transformations $x$ was computed in [24] (including the resonance terms of $t^{(1 s)}$ )

$$
\begin{aligned}
\hat{x}(v) & =x(v)+\mathcal{R}_{1 s}-t^{(1 s)} \\
& =\left(\frac{v_{0}^{s+1}}{(s+1) N_{s}}\right)\left(\frac{P_{s+1}(z)-P_{s-1}(z)}{2 s+1}\right)-\mathcal{R}_{1 n}\left(\frac{v_{0}^{s-n}}{N_{s-n}}\right) P_{s-n}(z)+O\left(\mathcal{R}^{2}\right),
\end{aligned}
$$

where $P_{n}(z)$ are Legendre polynomials with $z=v / v_{0}$ and $v_{0}=\sqrt{\mathcal{R}_{(11)} \frac{2(2 s-1)}{s(s+1)}}$. Here $N_{k}=2^{k} \Gamma(k+1 / 2) /(\Gamma(k+1) \Gamma(1 / 2))$ is a nomalization factor so that $\frac{v_{0}^{k}}{N_{k}} P_{k}(z)=v^{k}+\cdots$ is a monic polynomial.

The bulk generating function on the sphere is defined as

$$
\mathcal{F}(t)=\frac{1}{2} \int_{0}^{v_{0}} d v\left(\frac{\partial S}{\partial v}\right)^{2}
$$


Note that $v^{*}= \pm v_{0}$ since $\left.x\left( \pm v_{0}\right)\right|_{0}=0$. Here we choose $v^{*}=v_{0}$. The one-point function $\left\langle O_{1, n}\right\rangle$ (for $2 \leq n \leq s-1$ ) is given as

$$
\frac{\partial \mathcal{F}(t)}{\partial \mathcal{R}_{1, n}} \sim \int_{0}^{1} d z P_{s-n}(z)\left(\frac{P_{s+1}(z)-P_{s-1}(z)}{2 s+1}\right),
$$

which vanishes due to the orthogonality of the Legendre polynomials. In the same way, two-point correlation satisfies the orthogonality.

On the disk, the generating function $\mathcal{W}(l)$ in $(2.25)$ is used. However, we need a systematic way to find the perturbative contribution using the expression (4.5). For this purpose, we interpret $\mathcal{W}(l)$ as

$$
\mathcal{W}(l)=\int_{\mathcal{R}_{1 s}} d x_{0} \int_{i \mathbb{R}} d y e^{l\left(y^{2}+v\right)},
$$

where we use the notation $x_{0}=\left.\hat{x}(v)\right|_{\mathcal{R}_{1 n}=0}$ in (4.5)

$$
x_{0}=\left(\frac{v_{0}^{s+1}}{(s+1) N_{s}}\right)\left(\frac{P_{s+1}(z)-P_{s-1}(z)}{2 s+1}\right) .
$$

Then, the perturbative contribution is due to $\delta \mathcal{R}_{1 n}$ in (4.5) and its effect on $\delta v$ is calculated by constraining $\delta \hat{x}=0$.

The correlation $\left\langle O_{1, s}\right\rangle_{D}$ is simply given as

$$
\left\langle O_{1, s}\right\rangle_{D}=-\int_{i \mathbb{R}} d y e^{l\left(y^{2}+v^{*}\right)}=-\sqrt{\frac{\pi}{l}} e^{-l v_{0}} \propto \kappa^{1 / 2} K_{1 / 2}(\kappa l),
$$

where $\kappa=v_{0}$. We use $v^{*}=-v_{0}$ to make the integral convergent.

Other correlations $\left\langle O_{1, n \neq 1}\right\rangle_{D}$ are given as

$$
\left\langle O_{1, n}\right\rangle_{D}=\sqrt{\pi l} \int_{0} \mathrm{~d} x_{0}\left(\frac{\delta v}{\delta \mathcal{R}_{1, n}}\right)_{0} e^{l v} .
$$

The variation of $v$ is obtained from (4.5):

$$
\frac{\delta x_{0}}{\delta \mathcal{R}_{1 n}}=\frac{d x_{0}}{d v} \frac{\delta v}{\delta \mathcal{R}_{1 n}}=\left(\frac{v_{0}^{s-n}}{N_{s-n}}\right) P_{s-n}\left(v / v_{0}\right) .
$$

Therefore, the correlation is given as

$$
\left\langle O_{1, n}\right\rangle_{D}=\sqrt{\pi l}\left(\frac{v_{0}^{s-n}}{N_{s-n}}\right) \int_{-v_{0}}^{-\infty} \mathrm{d} v P_{s-n}\left(v / v_{0}\right) e^{l v} \sim \kappa^{s-n+1 / 2} K_{s-n+1 / 2}(l \kappa),
$$

where the integration formula is used:

$$
\int_{1}^{\infty} \mathrm{d} x P_{n}(x) e^{-y x}=\sqrt{\frac{2}{\pi y}} K_{n+1 / 2}(y) .
$$

The one-point correlations on the disk agree with FZZ result [39]. 


\section{$4.2 \mathrm{M}(3 / 5)$ : new features}

The first non-trivial non-unitary example is $\mathrm{M}(3 / 5)$ gravity, which is based on $A_{2}$ Frobenius manifold. It is noted that $\mathrm{M}(3 / 5)$ gravity has very different features from the unitary case. For example, the variable $x$ vanishes at the lowest order of the perturbation. The integration path of $x$ cannot be chosen by putting $v_{2}=0$ since $x \propto v_{2}$ vanishes identically in this case. As a result, the general framework breaks in $\mathrm{M}(3 / 5)$ gravity. The purpose of this section is to check carefully how one can cure the breakdown case by case.

Douglas action of $\mathrm{M}(3 / 5)$ is given with deformed parameters:

$$
\begin{aligned}
S & =t_{1}^{(1,2)} v_{1}+t_{2}^{(1,1)} v_{2}-H, & H & =\theta_{2,2}-t_{1,1}^{(13)} \theta_{1,1}-t_{1,2}^{(14)} \theta_{1,2}, \\
\theta_{2,2} & =-v_{2}\left(v_{1}^{3}-3 v_{2}^{2}\right) / 18, & \theta_{1,1} & =v_{1} v_{2}, \quad \theta_{1,2}=\left(-v_{1}^{4}+18 v_{1} v_{2}^{2}\right) / 36 .
\end{aligned}
$$

The parameters have $\mathbb{Z}_{2}$ symmetry: $t_{1}^{(1,2)}=t_{1}^{(2,3)}, t_{2}^{(1,1)}=t^{(2,4)}, t_{1,1}^{(13)}=t_{1,1}^{(22)}, t_{1,2}^{(14)}=t_{1,2}^{(21)}$, and g-dim is assigned as follows:

$$
\left[t_{1}^{(12)}\right]=7 / 6, \quad\left[t_{2}^{(11)}\right]=1, \quad\left[t_{1,1}^{(13)}\right]=4 / 6, \quad\left[t_{1,2}^{(14)}\right]=1 / 6 .
$$

The highest dimension is $7 / 6$ and therefore $[x]=\left[t_{1}\right]>[\mu]$. Instead, $\left[t_{2}^{(11)}\right]=1=\left[\mathcal{R}_{11}\right]$ and $\mathcal{R}_{11}=\mu$. According to g-dim analysis the following resonance transformations are allowed

$$
\begin{aligned}
& t_{1}^{(12)}=\mathcal{R}_{12}+A_{(11),(14)}^{(12)} \mu \mathcal{R}_{14}+O\left(\mathcal{R}^{4}\right), \quad t_{2}^{(11)}=\mathcal{R}_{11}+O\left(\mathcal{R}^{3}\right), \\
& t_{1,1}^{(13)}=\mathcal{R}_{13}+O\left(\mathcal{R}^{4}\right), \quad t_{1,2}^{(14)}=\mathcal{R}_{14} .
\end{aligned}
$$

The derivatives of the Douglas action have the form

$$
\begin{aligned}
& \frac{\partial S}{\partial v_{1}}=t^{(12)}-x ; \quad x=-v_{2} v_{1}^{2} / 6-t_{1,1}^{(13)} v_{2}-t_{1,2}^{(14)}\left(-v_{1}^{3} / 9+v_{2}^{2} / 2\right), \\
& \frac{\partial S}{\partial v_{2}}=t_{2}^{(11)}+\left(v_{1}^{3}-9 v_{2}^{2}\right) / 18+t_{1,1}^{(13)} v_{1}+t_{1,2}^{(14)} v_{1} v_{2} .
\end{aligned}
$$

On-shell solution is $v_{2}^{*}=0$ in (4.20) and $v_{1}^{* 3}=-18 \mu$ in (4.21), which looks the same as in the unitary series. However, on-shell solution $x^{*}$ vanishes unlike the unitary case.

Bulk generating function on the sphere is of the form (3.10). With on-shell solution the same as in the unitary series, one may check that the bulk one-point correlators $\left\langle O_{12}\right\rangle=$ $\left\langle O_{14}\right\rangle=0$ vanish since $v_{2}^{*}=0$. On the other hand, the gravitational dimension $\left[O_{13}\right]=$ 2 and $\left\langle O_{13}\right\rangle=0\left(\bmod \mu^{2}\right)$ as in $\mathrm{M}(3 / 4)$ case. Orthogonality of two-point correlations $\left\langle O_{12} O_{13}\right\rangle=0=\left\langle O_{13} O_{14}\right\rangle$ is fulfilled due to $v_{2}^{*}=0$. On the other hand, $\left\langle O_{12} O_{14}\right\rangle$ has additional contribution due to the resonance terms

$$
\left\langle O_{12} O_{14}\right\rangle=\int_{0}^{v_{1}^{*}} d v_{1}\left(-v_{1}^{3} / 9+A_{(11),(14)}^{(12)} \mu\right) .
$$

The orthogonality condition fixes the resonance coefficient:

$$
A_{(11),(14)}^{(12)} \mu=-v_{1}^{* 3} / 36 .
$$


For two-point correlations this requirement leads to the same results as in the unitary case: the resonance transformations are given in terms of Jacobi polynomials.

As in the Lee-Yang series, we use the generating function on the disk

$$
\mathcal{W}(l)=\int_{t_{1}^{(12)}} d x_{0} \int_{i \mathbb{R}} d y e^{l\left(y^{3}+t v_{1}+v_{2}\right)},
$$

with $x_{0}=-v_{2} v_{1}^{2} / 6$.

One-point correlator $\left\langle O_{1,2}\right\rangle_{D}$ is easily computed and has the form similar to (3.14)

$$
\left\langle O_{12}\right\rangle_{D}=-\int_{i \mathbb{R}} d y e^{l\left(y^{3}+v_{1}^{*} y\right)}=\frac{2 v_{1}^{* 1 / 2}}{3} K_{1 / 3}\left(2 l\left(v_{1}^{*} / 3\right)^{3 / 2}\right),
$$

where $v_{2}^{*}=0$ is used. This demonstrates that $\left\langle O_{1,2}\right\rangle_{D} \propto \kappa^{1 / 3} K_{1 / 3}(l \kappa)$.

Other correlators require more detailed information about $x_{0}$ and perturbation effects due to $\mathcal{R}_{1 k}$ 's. $\left\langle O_{11}\right\rangle_{D}$ is obtained by the perturbation of $\mathcal{R}_{11}$ :

$$
\left\langle O_{11}\right\rangle_{D}=\left.\int_{0}^{\infty} d x_{0} \int_{i \mathbb{R}} d y l\left(y \frac{\delta v_{1}}{\delta \mathcal{R}_{11}}+\frac{\delta v_{2}}{\delta \mathcal{R}_{11}}\right)_{0} e^{l\left(y^{3}+v_{1} y+v_{2}\right)}\right|_{0^{*}} .
$$

The variation of $v_{1}$ and $v_{2}$ due to $\mathcal{R}_{11}$ perturbation can be found from the string equation (4.21): $\delta v_{1} / \delta \mathcal{R}_{1,1}=-\left(6 / v_{1}^{2}\right)$ and $\delta v_{2} / \delta \mathcal{R}_{1,1}=0$. Therefore, we have

$$
\left\langle O_{11}\right\rangle_{D}=-\left.l \int_{0}^{\infty} d x_{0}\left(6 / v_{1}^{2}\right) \int_{i \mathbb{R}} d y y e^{l\left(y^{3}+v_{1} y+v_{2}\right)}\right|_{0^{*}} .
$$

Next step is to find the path of $x_{0}$-integration. Suppose we try to keep $v_{2}=0$ as in the unitary case. Then, one has $d x_{0}=-d v_{1}\left(v_{1} v_{2} / 3\right)$ and the integration has null value. This suggests that we cannot follow the general framework used in the unitary case.

Instead, we may prescribe the integration path alternatively: keep the contour path $v_{1}=v_{1}^{*}$ and $v_{2} \in[0, \infty]$. In this case, using $d x=-d v_{2}\left(v_{1}^{2} / 6\right)$ we have

$$
\left\langle O_{11}\right\rangle_{D}=\int_{0}^{-\infty} d v_{2}\left(-\frac{v_{1}^{* 2}}{6}\right) \int_{R} d y l y\left(-\frac{6}{v_{1}^{* 2}}\right) e^{i l\left(y^{3}+v_{1}^{*} y+v_{2}\right)} .
$$

The result is proportional to $\kappa^{2 / 3} K_{2 / 3}(l \kappa)$ as expected. Noting that the integrand is not closed, the contour integration depends on the path deformation. One needs proper integration path to achieve the right expectation value. Therefore, the choice of the integration contour looks an ad hoc prescription to get an expected answer.

It is not clear at this moment whether there exists any canonical choice of the integration path. We present further examples illustrating this problem. The similar phenomenon occurs when we consider $\left\langle O_{13}\right\rangle_{D}$. Varying the string equation we get

$$
\frac{\delta v_{1}}{\delta \mathcal{R}_{13}}=-6 / v_{1}, \quad \frac{\delta v_{2}}{\delta \mathcal{R}_{13}}=-6 v_{2} / v_{1}^{2} .
$$

Taking the same integration contour as in (4.28) we get

$$
\begin{aligned}
\left\langle O_{13}\right\rangle_{D} & =\int_{0}^{-\infty} d v_{2}\left(-\frac{v_{1}^{* 2}}{6}\right) \int_{i \mathbb{R}} d y l\left(\left(-\frac{6}{v_{1}^{* 2}} v_{2}\right)+y\left(-\frac{6}{v_{1}^{*}}\right)\right) e^{l\left(y^{3}+v_{1}^{*} y+v_{2}\right)} \\
& =\int_{i \mathbb{R}} d y\left(\frac{1}{l}-y v_{1}^{*}\right) e^{l\left(y^{3}+v_{1}^{*} y+v_{2}\right)} \propto \kappa^{4 / 3} K_{4 / 3}(l \kappa)
\end{aligned}
$$

which leads to the desired answer. 
One point function $\left\langle O_{14}\right\rangle_{D}$ represents more complicated case. Suppose we keep $v_{2}=0$. Then, the string equation shows that

$$
\frac{\delta v_{1}}{\delta \mathcal{R}_{14}}=0, \quad \frac{\delta v_{2}}{\delta \mathcal{R}_{14}}=\frac{2 v_{1}^{2}}{3} .
$$

Then the one-point function vanishes since the measure is proportional to $v_{2}$.

One possible way out is to interpret the generating function in a different way. Instead of using the variable $x_{0}$ in the generating function (4.24), we may use variable $x$ in defining the generating function

$$
\mathcal{W}(l)=\int_{t_{1}^{(12)}} d x \int_{i \mathbb{R}} d y e^{l\left(y^{3}+t v_{1}+v_{2}\right)}
$$

and allow the variation of the measure $d x$ while assuming $Q$ remains fixed so that

$$
\left\langle O_{14}\right\rangle_{D}=\int_{v_{1}^{*}}^{\infty} d v_{1}\left(\left(-\frac{v_{1}}{3}\right)\left(\frac{\delta v_{2}}{\delta \mathcal{R}_{14}}\right)+\frac{v_{1}^{2}}{3}\right) \int_{i \mathbb{R}} d y e^{l\left(y^{3}+v_{1} y\right)}+\frac{v_{1}^{* 3}}{36}\left\langle O_{1,2}\right\rangle_{D},
$$

which would lead to

$$
\begin{aligned}
\left\langle O_{14}\right\rangle_{D} & =\int_{v_{1}^{*}}^{\infty} d v_{1} \int_{i R} d y\left(\left(-\frac{v_{1}}{3}\right)\left(\frac{2 v_{1}}{3}\right)+\frac{v_{1}^{2}}{3}\right) e^{l\left(y^{3}+v_{1} y\right)}+\frac{v_{1}^{* 3}}{36}\left\langle O_{1,2}\right\rangle_{D} \\
& \propto \kappa^{7 / 3} K_{7 / 3}(l \kappa),
\end{aligned}
$$

in agreement with the FZZ result.

\section{Concluding remarks}

We use the matrix formalism to compute bulk correlations of minimal gravity on the sphere and on the disk and compare the result with the results of the Liouville gravity approach. We use the sphere correlation generating function proposed in [25] and describe the effect of the resonance transformations for computing bulk correlators in the presence of boundary. We clarify some subtleties in the construction of the generating function if one generalizes the matrix framework to $M(q / p)$ gravity, based on $A_{q-1}$ Frobenius manifold with $q>2$. We demonstrate that the matrix approach and the Liouville field approach agree with each other for the one and two correlations on the sphere and for the one-point correlation on the disk.

Even though the framework of the matrix approach looks similar for the unitary and non-unitary series, the details of the calculation show some subtleties. In the unitary series $M(q / q+1)$, continuous variable $x$ of the matrix model approach has the same gravitational scaling dimension as the cosmological constant $\mu$. For the non-unitary series, the gravitational dimension of $x$ is greater than that of cosmological constant. On the sphere, however, this subtlety is hidden because $x$ does not come explicitly in the expression for the generating function. On the other hand, the computation of the bulk correlation on the disk requires knowledge of the specific dependence of $x$ on the flat coordinates according to the Douglas string equation. We provide some details of unitary series in 
section 3 and of non-unitary series in section 4 and find the consistency of the formalism once the subtleties are well taken care of.

It is noted that on-shell condition defined in section 2.2, which is equivalent to a specific solution to the Douglas equation, is enough to find the correlation on the disk for unitary series, whose calculation is simplified in the flat coordinates of the Frobenius manifold. For the non-unitary Lee-Yang series $M(2 / 2 s+1)$, the Frobenius manifold is one dimensional $A_{1}$ and hence is trivial. In this case, one can simply use the off-shell condition for the flat coordinate. However, for higher $q$ non-unitary models, when the Frobenius manifold becomes multi-dimensional, the usage of the matrix model results requires more profound analysis of the off-shell condition. The considered examples show that there is no canonical way of evaluating the one-point correlation on the disk for the non-unitary models without using some additional assumptions. Even though there are certain conjectures about the choice of the contours and the off-shell application of the string equation leading to the desired FZZ result, the analytic structure of the generating function on the disk is not fully understood and needs further investigation.

Among other possible further developments of the present analysis we mention the following natural questions. The case of non-trivial boundary conditions as well as of the other types of correlations involving boundary insertions remains to be studied. In particular, the resonance transformations in this case can be affected by the presence of boundary fields and further analysis of the Frobenius manifold structure hidden beyond the matrix model formulation is required. In this context the analysis of the connection between the intersection theory on the moduli space of Riemann surfaces with boundary and the open KdV equations might be useful (see, e.g. [57-60]). Finally, we note that the new methods of the dual approach to the minimal (open and closed) strings based on the connection with the theory of Frobenius manifolds suggests that maybe some of these results can be applied for analytic computations of string amplitudes in more realistic string models. In this respect the consideration in [61], where $c=1$ string theory has been considered from the worldsheet perspective and compared with the matrix models, may be relevant.

\section{Acknowledgments}

The work of K.A. and V.B. was supported by the Foundation for the advancement of theoretical physics "BASIS". The work of C.R. was partially supported by National Research Foundation of Korea grant NRF-2017R1A2A2A05001164.

\section{A Bessel functions and Chebyshev polynomials}

Below we list some useful formulae for Bessel K-functions and Chebyshev polynomials

$$
\begin{aligned}
& K_{\nu}(z)=\int_{0}^{\infty} \mathrm{d} s \operatorname{ch}(\nu s) e^{-z \operatorname{ch}(s)}, \\
& K_{\nu}(z)=\frac{1}{\cos (\nu \pi / 2)} \int_{0}^{\infty} \mathrm{d} s \operatorname{ch}(\nu s) \cos (z \operatorname{sh}(s)),
\end{aligned}
$$




$$
\begin{aligned}
K_{\nu}(z) & =\frac{1}{\sin (\nu \pi / 2)} \int_{0}^{\infty} \mathrm{d} s \operatorname{sh}(\nu s) \sin (z \operatorname{sh}(s)), & & \\
T_{n}(i \operatorname{sh}(s)) & =(-1)^{(n-1) / 2} i \operatorname{sh}(n s), & & n=2 k-1, \\
T_{n}(i \operatorname{sh}(s)) & =(-1)^{n / 2} \operatorname{ch}(n s), & & n=2 k .
\end{aligned}
$$

To perform the $x$ integration we use:

$$
\begin{aligned}
\int_{\mu}^{\infty} \mathrm{d} x x^{\nu / 2} K_{\nu}\left(x^{1 / 2}\right) & =2 \mu^{(\nu+1) / 2} K_{\nu+1}\left(\mu^{1 / 2}\right), \\
K_{\nu}(x) & =K_{-\nu}(x), \\
K_{\nu}(x) & =\frac{x}{2 \nu}\left(K_{\nu+1}(x)-K_{\nu-1}(x)\right) .
\end{aligned}
$$

In particular, for $M(3, p)$ models we have

$$
\int_{\mathbb{R}} \mathrm{d} y e^{i l\left(y^{3}+v_{1} y\right)}=\frac{2 v_{1}^{1 / 2}}{3} K_{1 / 3}\left(2 l\left(v_{1} / 3\right)^{3 / 2}\right) .
$$

Open Access. This article is distributed under the terms of the Creative Commons Attribution License (CC-BY 4.0), which permits any use, distribution and reproduction in any medium, provided the original author(s) and source are credited.

\section{References}

[1] A.M. Polyakov, Quantum Geometry of Bosonic Strings, Phys. Lett. B 103 (1981) 207 [INSPIRE].

[2] A.A. Belavin, A.M. Polyakov and A.B. Zamolodchikov, Infinite Conformal Symmetry in Two-Dimensional Quantum Field Theory, Nucl. Phys. B 241 (1984) 333 [inSPIRE].

[3] H. Dorn and H.-J. Otto, On correlation functions for noncritical strings with $c \leqslant 1$ but $d \geqslant 1$, Phys. Lett. B 291 (1992) 39 [hep-th/9206053] [INSPIRE].

[4] A.B. Zamolodchikov and A.B. Zamolodchikov, Structure constants and conformal bootstrap in Liouville field theory, Nucl. Phys. B 477 (1996) 577 [hep-th/9506136] [INSPIRE].

[5] F. David, A. Kupiainen, R. Rhodes and V. Vargas, Liouville Quantum Gravity on the Riemann sphere, Commun. Math. Phys. 342 (2016) 869 [arXiv:1410.7318] [InSPIRE].

[6] A. Kupiainen, R. Rhodes and V. Vargas, Integrability of Liouville theory: proof of the DOZZ Formula, arXiv: 1707.08785 [INSPIRE].

[7] A.A. Belavin and A.B. Zamolodchikov, Integrals over moduli spaces, ground ring and four-point function in minimal Liouville gravity, Theor. Math. Phys. 147 (2006) 729 [INSPIRE].

[8] K. Aleshkin and V. Belavin, On the construction of the correlation numbers in Minimal Liouville Gravity, JHEP 11 (2016) 142 [arXiv:1610.01558] [INSPIRE].

[9] V.A. Kazakov, A.A. Migdal and I.K. Kostov, Critical Properties of Randomly Triangulated Planar Random Surfaces, Phys. Lett. B 157 (1985) 295 [inSPIRE]. 
[10] V.A. Kazakov, Ising model on a dynamical planar random lattice: Exact solution, Phys. Lett. A 119 (1986) 140 [inSPIRE].

[11] V.G. Knizhnik, A.M. Polyakov and A.B. Zamolodchikov, Fractal Structure of $2 D$ Quantum Gravity, Mod. Phys. Lett. A 3 (1988) 819 [InSPIRE].

[12] M.R. Douglas, Strings in Less Than One-dimension and the Generalized KdV Hierarchies, Phys. Lett. B 238 (1990) 176 [INSPIRE].

[13] E. Witten, Two-dimensional gravity and intersection theory on moduli space, Surveys Diff. Geom. 1 (1991) 243 [INSPIRE].

[14] R. Dijkgraaf, H.L. Verlinde and E.P. Verlinde, Loop equations and Virasoro constraints in non-perturbative two-dimensional quantum gravity, Nucl. Phys. B 348 (1991) 435 [INSPIRE].

[15] M. Kontsevich, Intersection theory on the moduli space of curves and the matrix Airy function, Commun. Math. Phys. 147 (1992) 1 [INSPIRE].

[16] V.A. Kazakov, The Appearance of Matter Fields from Quantum Fluctuations of $2 D$ Gravity, Mod. Phys. Lett. A 4 (1989) 2125 [INSPIRE].

[17] M. Staudacher, The Yang-Lee Edge Singularity on a Dynamical Planar Random Surface, Nucl. Phys. B 336 (1990) 349 [InSPIRE].

[18] M.R. Douglas and S.H. Shenker, Strings in Less Than One-Dimension, Nucl. Phys. B 335 (1990) 635 [inSPIRE].

[19] D.J. Gross and A.A. Migdal, Nonperturbative Two-Dimensional Quantum Gravity, Phys. Rev. Lett. 64 (1990) 127 [INSPIRE].

[20] E. Brézin and V.A. Kazakov, Exactly Solvable Field Theories of Closed Strings, Phys. Lett. B 236 (1990) 144 [INSPIRE].

[21] G.W. Moore, N. Seiberg and M. Staudacher, From loops to states in two-dimensional quantum gravity, Nucl. Phys. B 362 (1991) 665 [INSPIRE].

[22] A.B. Zamolodchikov, Higher equations of motion in Liouville field theory, Int. J. Mod. Phys. A 19S2 (2004) 510 [hep-th/0312279] [INSPIRE].

[23] A.A. Belavin and A.B. Zamolodchikov, Integrals over moduli spaces, ground ring and four-point function in minimal Liouville gravity, Theor. Math. Phys. 147 (2006) 729 [INSPIRE].

[24] A.A. Belavin and A.B. Zamolodchikov, On Correlation Numbers in 2D Minimal Gravity and Matrix Models, J. Phys. A 42 (2009) 304004 [arXiv:0811.0450] [INSPIRE].

[25] A. Belavin, B. Dubrovin and B. Mukhametzhanov, Minimal Liouville Gravity correlation numbers from Douglas string equation, JHEP 01 (2014) 156 [arXiv:1310.5659] [INSPIRE].

[26] V. Belavin, Unitary Minimal Liouville Gravity and Frobenius Manifolds, JHEP 07 (2014) 129 [arXiv: 1405.4468] [INSPIRE].

[27] A.A. Belavin and V.A. Belavin, Frobenius manifolds, Integrable Hierarchies and Minimal Liouville Gravity, JHEP 09 (2014) 151 [arXiv: 1406.6661] [INSPIRE].

[28] V. Belavin, Correlation Functions in Unitary Minimal Liouville Gravity and Frobenius Manifolds, JHEP 02 (2015) 052 [arXiv: 1412.4245] [INSPIRE].

[29] V. Belavin and Y. Rud, Matrix model approach to minimal Liouville gravity revisited, J. Phys. A 48 (2015) 18FT01 [arXiv:1502.05575] [InSPIRE]. 
[30] B. Dubrovin, Integrable systems in topological field theory, Nucl. Phys. B 379 (1992) 627 [INSPIRE].

[31] G. Tarnopolsky, Five-point Correlation Numbers in One-Matrix Model, J. Phys. A 44 (2011) 325401 [arXiv:0912.4971] [InSPIRE].

[32] A. Belavin, M. Bershtein and G. Tarnopolsky, A remark on the three approaches to $2 D$ Quantum gravity, JETP Lett. 93 (2011) 47 [arXiv: 1010.2222] [INSPIRE].

[33] V. Belavin, Torus Amplitudes in Minimal Liouville Gravity and Matrix Models, Phys. Lett. B 698 (2011) 86 [arXiv: 1010.5508] [INSPIRE].

[34] L. Spodyneiko, Minimal Liouville gravity on the torus via the Douglas string equation, J. Phys. A 48 (2015) 065401 [inSPIRE].

[35] J.L. Cardy, Conformal Invariance and Surface Critical Behavior, Nucl. Phys. B 240 (1984) 514 [inSPIRE].

[36] J.L. Cardy, Effect of Boundary Conditions on the Operator Content of Two-Dimensional Conformally Invariant Theories, Nucl. Phys. B 275 (1986) 200 [INSPIRE].

[37] J.L. Cardy and D.C. Lewellen, Bulk and boundary operators in conformal field theory, Phys. Lett. B 259 (1991) 274 [INSPIRE].

[38] I. Runkel, Boundary structure constants for the A-series Virasoro minimal models, Nucl. Phys. B 549 (1999) 563 [hep-th/9811178] [INSPIRE].

[39] V. Fateev, A.B. Zamolodchikov and A.B. Zamolodchikov, Boundary Liouville field theory. 1. Boundary state and boundary two point function, hep-th/0001012 [INSPIRE].

[40] B. Ponsot and J. Teschner, Boundary Liouville field theory: Boundary three point function, Nucl. Phys. B 622 (2002) 309 [hep-th/0110244] [INSPIRE].

[41] I.K. Kostov, B. Ponsot and D. Serban, Boundary Liouville theory and 2D quantum gravity, Nucl. Phys. B 683 (2004) 309 [hep-th/0307189] [INSPIRE].

[42] J.-E. Bourgine, K. Hosomichi and I.K. Kostov, Boundary transitions of the $O(n)$ model on a dynamical lattice, Nucl. Phys. B 832 (2010) 462 [arXiv:0910.1581] [INSPIRE].

[43] J.-E. Bourgine and K. Hosomichi, Boundary operators in the $O(n)$ and RSOS matrix models, JHEP 01 (2009) 009 [arXiv:0811.3252] [INSPIRE].

[44] I.K. Kostov, Boundary correlators in $2 D$ quantum gravity: Liouville versus discrete approach, Nucl. Phys. B 658 (2003) 397 [hep-th/0212194] [INSPIRE].

[45] J.L. Jacobsen and H. Saleur, Conformal boundary loop models, Nucl. Phys. B 788 (2008) 137 [math-ph/0611078] [INSPIRE].

[46] G. Ishiki and C. Rim, Boundary correlation numbers in one matrix model, Phys. Lett. B 694 (2011) 272 [arXiv:1006.3906] [INSPIRE].

[47] E.J. Martinec, G.W. Moore and N. Seiberg, Boundary operators in 2D gravity, Phys. Lett. B 263 (1991) 190 [inSPIRE].

[48] K. Hosomichi, Minimal Open Strings, JHEP 06 (2008) 029 [arXiv:0804.4721] [inSPIRE].

[49] A. Belavin and C. Rim, Bulk one-point function on disk in one-matrix model, Phys. Lett. B 687 (2010) 264 [arXiv:1001.4356] [INSPIRE].

[50] A.A. Belavin and A.B. Zamolodchikov, On Correlation Numbers in 2D Minimal Gravity and Matrix Models, J. Phys. A 42 (2009) 304004 [arXiv:0811.0450] [InSPIRE]. 
[51] J.-E. Bourgine, G. Ishiki and C. Rim, Bulk-boundary correlators in the hermitian matrix model and minimal Liouville gravity, Nucl. Phys. B 854 (2012) 853 [arXiv:1107.4186] [INSPIRE].

[52] P.H. Ginsparg and G.W. Moore, Lectures on $2 D$ gravity and $2 D$ string theory, in proceedings of the Theoretical Advanced Study Institute (TASI 92): From Black Holes and Strings to Particles, Boulder, Colorado, U.S.A., 3-28 June 1992, pp. 277-469 [hep-th/9304011] [INSPIRE].

[53] P. Di Francesco, P.H. Ginsparg and J. Zinn-Justin, 2D Gravity and random matrices, Phys. Rept. 254 (1995) 1 [hep-th/9306153] [INSPIRE].

[54] P.H. Ginsparg, M. Goulian, M.R. Plesser and J. Zinn-Justin, $(p, q)$ string actions, Nucl. Phys. B 342 (1990) 539 [INSPIRE].

[55] N. Seiberg and D. Shih, Branes, rings and matrix models in minimal (super)string theory, JHEP 02 (2004) 021 [hep-th/0312170] [INSPIRE].

[56] P. Di Francesco and D. Kutasov, Integrable Models of Two Dimensional Quantum Gravity, Springer, Boston Massachusetts U.S.A. (1991), pp. 35-51.

[57] R. Pandharipande, J.P. Solomon and R.J. Tessler, Intersection theory on moduli of disks, open KdV and Virasoro, arXiv:1409.2191 [INSPIRE].

[58] A. Buryak, Equivalence of the open KdV and the open Virasoro equations for the moduli space of Riemann surfaces with boundary, Lett. Math. Phys. 105 (2015) 1427 [arXiv: 1409.3888] [INSPIRE].

[59] A. Buryak, Open intersection numbers and the wave function of the KdV hierarchy, Moscow Math. J. 16 (2016) 27 [arXiv: 1409.7957] [INSPIRE].

[60] M. Bertola and D. Yang, The partition function of the extended r-reduced Kadomtsev-Petviashvili hierarchy, J. Phys. A 48 (2015) 195205 [arXiv:1411.5717] [INSPIRE].

[61] B. Balthazar, V.A. Rodriguez and X. Yin, The $c=1$ String Theory S-matrix Revisited, arXiv: 1705.07151 [INSPIRE]. 